IAC-09.D1.6.8

\title{
DYNAMIC SYSTEM SIMULATION OF SMALL SATELLITE PROJECTS
}

\author{
Matthias Raif, Ulrich Walter \\ TU Muenchen, Institute of Astronautics, Garching, Germany \\ m.raif@tum.de, u.walter@tum.de \\ Jasper Bouwmeester \\ Delft University of Technology, Chair of Space Systems Engineering, Delft, Netherlands \\ jasper.bouwmeester@tudelft.nl
}

\begin{abstract}
A prerequisite to accomplish a system simulation is to have a system model holding all necessary project information in a centralized repository that can be accessed and edited by all parties involved. At the Institute of Astronautics of the Technische Universitaet Muenchen a modular approach for modeling and dynamic simulation of satellite systems has been developed called Dynamic System Simulation (DySyS). DySyS is based on the platform independent description language SysML to model a small satellite project with respect to the system composition and dynamic behavior. A library of specific building blocks and possible relations between these blocks have been developed. From this library a system model of the satellite of interest can be created. A mapping into a C++ simulation allows the creation of an executable system model with which simulations are performed to observe the dynamic behavior of the satellite. In this paper DySyS is used to model and simulate the dynamic behavior of small satellites, because small satellite projects can act as a precursor to demonstrate the feasibility of a system model since they are less complex compared to a large scale satellite project
\end{abstract}

\section{INTRODUCTION}

A dynamic system simulation can greatly improve the development process of a satellite. Computational simulations can be performed in an early stage of the development process to gain deeper insights into the system performance. Without having any hardware under test, design flaws can be detected and eradicated. With a dynamic simulation it is possible to test the spacecraft design with respect to the requirements to ensure that the performance will satisfy all needs. As a predictive tool a system simulation can be used to evaluate different design alternatives in a trade study. It also can be used to size components of the satellite's subsystems. By varying the parameter in question several simulations can be made. The results of the different simulations can be compared and conclusions are drawn to derive the best design.

System simulation is a very special form of simulation. It incorporates all the different engineering disciplines into one system model. This model is used in a simulation to analyze the behavior of the complete system. During satellite development a wide range of simulation tools is available to conduct simulations for any special purpose, as there are for example: mission analysis and orbit simulation, structural and thermal analysis, or attitude and control simulation. All these tools are specialized on a certain aspect of the system. None of these tools is able to model and simulate the entire system even though many system details are similar in these separated simulations. In contrast a system simulation will incorporate many aspects into one simulation. Thus it comprises the interactions and influences between the different system aspects and its corresponding dynamic behavior. The drawback of a system simulation is the complexity of the model. To reduce computational efforts one has to reduce the fidelity and depth of model detail in some aspects compared to dedicated simulation tools. So system simulation cannot completely substitute all other simulations but it can certainly contribute as a valuable supplement.

In this paper we concentrate on the use of dynamic system simulation for small satellite projects, especially in the nanosatellite size. The method described is used to predict the system performance in the early design phase of the project. Therefore it uses simple models to limit complexity. The method is modular and adaptable enough that it can also be used in later phases. The modularity also permits to reuse the developed models in other projects. In fact special attention was paid to the modularity and reusability of the model. In the future there shall be a comprehensive library of building blocks from which a satellite project can be virtually assembled and simulated. 


\section{SYSTEM DYNAMIC}

The purpose of a dynamic system simulation is to predict or validate the dynamic behavior of a system. This is done by running on a system model representing the real system on a computer. The dynamic behavior is defined as the evolution of all system states over time. The system model has to reproduce this temporal change in an appropriate way. Certainly not all real effects can be included in the system model. Simplifications and abstraction have to be made. To evaluate the system performance of a spacecraft design the most important dynamic behaviors are the kinetic and attitude behavior, the electrical behavior (generation, distribution, and consumption of electrical energy), the thermal behavior (temperature distribution) and the communication behavior (inside and outside the spacecraft). The dynamic behavior of a spacecraft is best described by differential equations specifying the change of system states over time. For the dynamic simulation these differential equations have to be solved and evaluated. This is done by numerical methods.

As only the overall dynamic behavior of the spacecraft system is of interest some simplifications on the model are made:

- Only simple differential equations are evaluated. No partial differential equations are used.

- Forces on structural elements cause no deformation.

- No strain or deformation due to thermal effects.

- Hard radiation and their effects on electrical devices are not modeled (except for solar cells).

- Electromagnetic compatibility of electrical devices is not considered.

- Only Earth orbiting satellites can be simulated at the moment (due to the chosen coordinate frame).

\section{$\underline{\text { Kinetic and attitude behavior }}$}

The movement and pointing of the spacecraft is evaluated with the help of two coordinate systems.

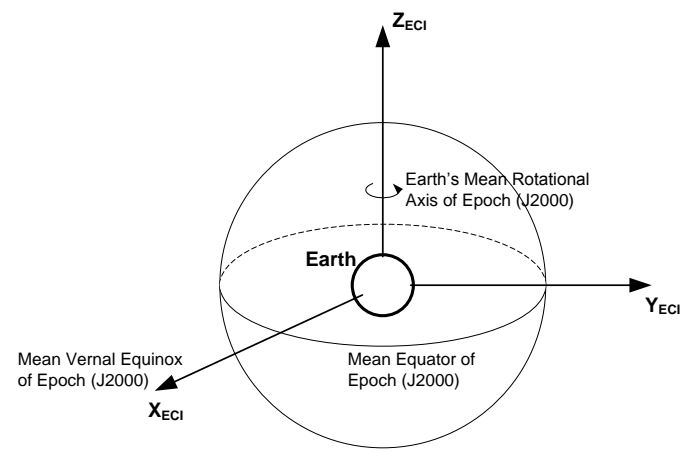

Fig. 1: Definition of the Earth centered inertial coordinate system (ECI).
The system-states position and velocity are given in the Earth centered inertial coordinate system (ECI) (see Fig. 1). The origin of the ECI coordinate system is located at the Earth's center. The X-axis lies on the intersection of equatorial plane and the ecliptic, pointing to the First Point of Aries. The z-axis lies on Earth's rotational axis pointing northwards. The yaxis completes the coordinate system to a right handed one.

The rate of change of the spacecraft's position $\boldsymbol{r}$ and velocity $v$ can be expressed by two ordinary differential equations of first order:

$$
\begin{gathered}
\frac{d \boldsymbol{v}}{d t}=\boldsymbol{a}_{\text {extern }}+\frac{\boldsymbol{F}_{\text {total }}}{m_{\text {system }}} \\
\frac{d \boldsymbol{r}}{d t}=\boldsymbol{v} .
\end{gathered}
$$

Here $\boldsymbol{a}_{\text {extern }}$ are the external accelerations acting on the system, $\boldsymbol{F}_{\text {total }}$ is the sum of all acting forces and $m_{\text {system }}$ is the mass of the system.

The system-state attitude is given as a quaternion describing the orientation of the body fixed coordinate system with respect to the inertial coordinate system. The system state angular velocity is given as the angular rates about these body axes.

The rotational movement is described by the rate of change of the angular velocity $\omega$

$$
\frac{d \boldsymbol{\omega}}{d t}=\boldsymbol{I}^{-1}\left(\boldsymbol{T}_{\text {total }}-\boldsymbol{\omega} \times \boldsymbol{h}_{\text {total }}\right)
$$

with $\boldsymbol{I}$ as the moment of inertia of the rigid body, the sum of all acting torques $\boldsymbol{T}_{\text {total }}$ and the total angular momentum $\boldsymbol{h}_{\text {total }}$ of the system. The rate of change of attitude is given by

$$
\begin{gathered}
\frac{d \boldsymbol{q}}{d t}=\frac{1}{2} \boldsymbol{\Omega} \boldsymbol{q} \\
\boldsymbol{\Omega}=\left[\begin{array}{cccc}
0 & -\omega_{1} & -\omega_{2} & -\omega_{3} \\
\omega_{1} & 0 & \omega_{3} & -\omega_{2} \\
\omega_{2} & -\omega_{3} & 0 & \omega_{1} \\
\omega_{3} & \omega_{2} & -\omega_{1} & 0
\end{array}\right]
\end{gathered}
$$

with the quaternion $\boldsymbol{q}$ and the angular velocity matrix $\Omega$.

\section{Electrical behavior}

The electrical behavior describes the generation, storage and consumption of electrical energy inside the spacecraft.

Most of all spacecraft rely on solar cells for the generation of electrical energy. The production rate of solar cells is mainly dependent on the incidence angle of the incoming sunlight, i.e. the attitude of the spacecraft.

The storage of electrical energy is solved by chemo-electrical batteries. The rate of change of current capacity of a battery can be described by a simple differential equation. 


$$
\frac{d Q}{d t}=P_{\text {total }}
$$

with $Q$ as the stored electrical energy and $P_{\text {total }}$ as the sum of all generated and consumed electrical power, including all conversion losses on the path.

\section{Thermal behavior}

The thermal behavior describes the temperature distribution inside the spacecraft. As we want to pass on partial differential equations only a lumped parameter model comes into consideration. Here several isothermal nodes exchange thermal energy by conduction and radiation. The number of nodes should be limited to minimize computational effort.

The conductive heat exchange on one node $i$ is described by

$$
\dot{Q_{c}}=\sum_{j} C_{i j}\left(T_{j}-T_{i}\right)
$$

the radiation exchange by

$$
\dot{Q}_{r}=\sigma \sum_{j} R_{i j}\left(T_{j}^{4}-T_{i}^{4}\right)
$$

where $\sigma$ is the Stefan-Boltzmann constant.

A hotter neighboring node $j\left(T_{j}>T_{i}\right)$ results in a positive heat flow on node $i . C_{i j}$ describe the conductive heat-transfer coefficients, $R_{i j}$ the radiation heat-transfer coefficients between $i$ and $j$.

Each node is characterized by its temperature (the state) and the heat capacity. The rate of change of the temperature is given by

$$
\frac{d T}{d t}=\frac{1}{m \cdot c} \dot{Q}_{\text {total }}
$$

where $m$ is the mass of the node and $c$ the specific heat capacity. $\dot{Q}_{\text {total }}$ is the sum of all heat flows acting on that node

$$
\dot{Q}_{\text {total }}=\dot{Q}_{c}+\dot{Q}_{r}+\dot{Q}_{\text {diss }}
$$

with $\dot{Q}_{\text {diss }}$ as the dissipated heat (e.g. electrical conversion losses) inside the node.

\section{Communication and data handling behavior}

A spacecraft can be seen as an embedded system. A lot of sensors and actuators communicate with an onboard computer via special data handling protocols. For the dynamic system simulation, these data handling have to be modeled only if hardwarein-the-loop tests are involved. In early phases of system design, these analyses can be discarded.

The communication between the spacecraft and the ground station are of interest, especially the quality of the connection and its time of availability. These calculations are highly dependent on the position and attitude of the spacecraft in relation to the ground station.

\section{$\underline{\text { SYSTEM MODELING }}$}

The system model for the dynamic simulation should be built very modular to achieve reusability of the model. Each modular element of the model represents a certain part of the system. The element is defined in a generic way so it can represent different types of characteristics. The definition of an element includes the definition of parameters, interfaces and methods. Interaction between elements is done by relations which can exchange a specified type of item (such as energy, mass, force, data, etc.).

A generic model library of elements is created in a platform independent way using SysML as description language [1]. The modeling method uses the concept of inheritance (Fig. 2) to build up a hierarchy in the model library [2].

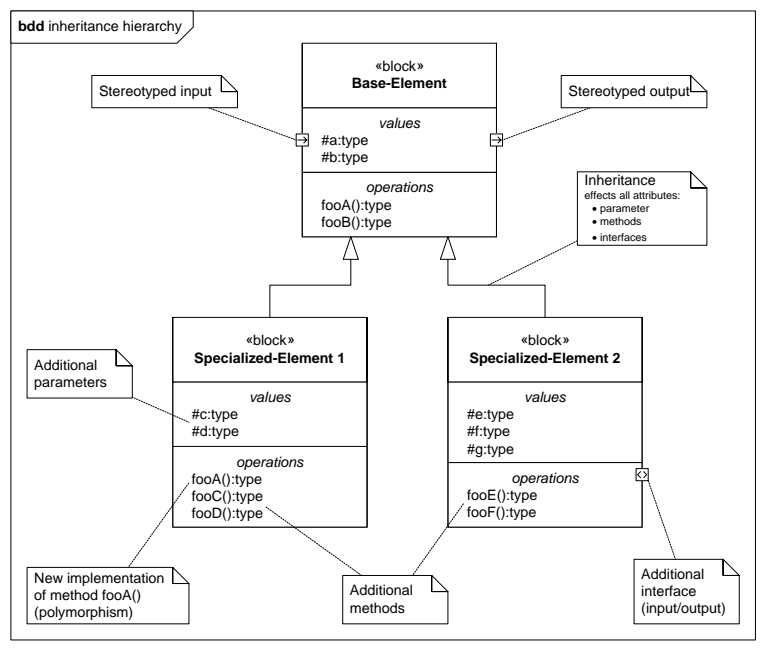

Fig. 2: Concept of inheritance in the element library.

All elements in the system model are derived from three basic element classes: the system class, the subsystem class and the device class.

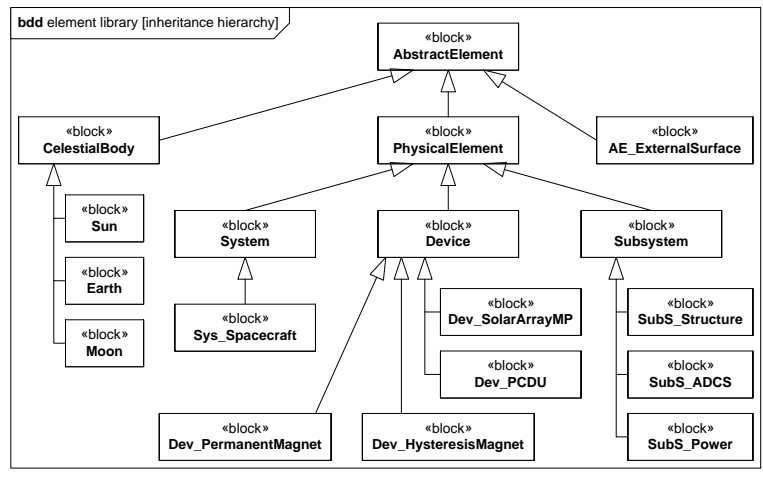

Fig. 3: Complete inheritance diagram of the used library element classes. 
These basic elements also define the system model hierarchy formed by composition relations. A system model is created by decomposing the system into element classes that are described in the library. The complete inheritance hierarchy of the used element classes can be seen in Fig. 3 .

\section{MODEL LIBRARY}

The following paragraphs give a detailed explanation on the element classes used in this paper. The SysML descriptions shown here are shortened to the structural definition only. The functionality and behavior is explained in the text and with formulas. In the developed modeling method this information is also described by SysML diagrams (parametric diagram, state chart diagram).

\section{Environment}

The environment of a spacecraft is mainly defined by the celestial bodies in its vicinity. Three different celestial bodies have been considered: The Sun, the Earth and the Moon. All three bodies are based on a common more general element class, in which all parameters, interfaces and methods are declared. The specific characteristic is done in the three specialized element classes (see Fig. 4).

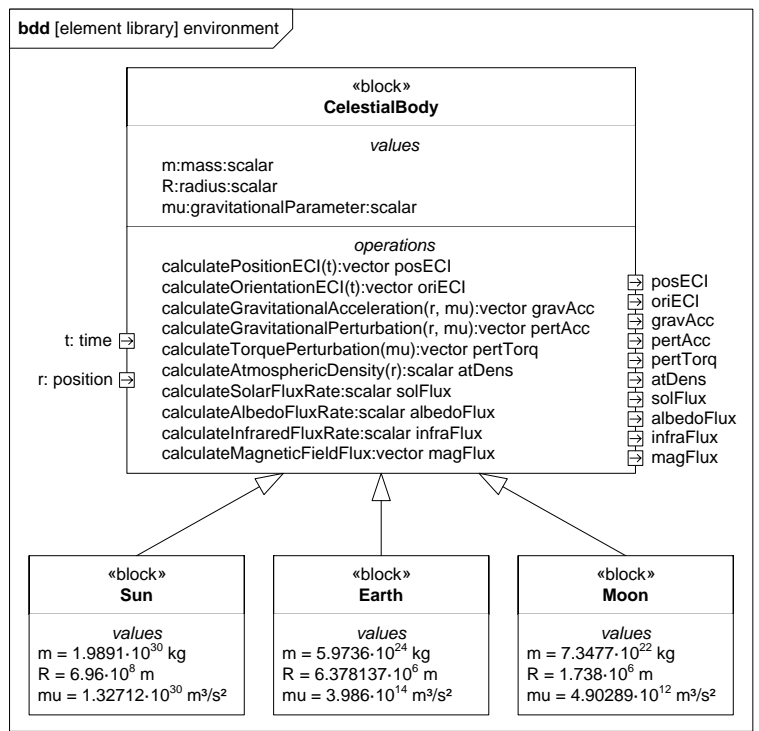

Fig. 4: Definition of the element class CelestialBody.

The state of the celestial bodies, i.e. their position and attitude in the ECI coordinate system is determined by approximations given in [3].

The influence of a celestial body on the kinetics of a spacecraft is mainly given by gravitational effects. The gravitational attraction on a spacecraft can be divided into separate fractions. The main attraction of a central celestial body is dependent on the distance and can be calculated as acceleration by

$$
\boldsymbol{a}_{\mathrm{grav}}=-\boldsymbol{r} \frac{\mu}{|\boldsymbol{r}|^{3}}
$$

where $\boldsymbol{r}$ is the radius vector from the celestial body center and $\mu$ its gravitational parameter. In addition to this main attraction, some perturbations have to be considered. For a spacecraft in Earth orbit the variations of Earth's gravitational field can be calculated by a spherical harmonic expansion term $B$ as part of the total gravitational potential $U$. The acceleration is obtained by determining the gradient of the gravitational potential $\nabla U$. In spherical coordinates the perturbation part of Earth's gravitational potential can be expressed by

$$
\begin{aligned}
B(r, \theta, \phi)= & \frac{\mu_{\oplus}}{r}\left\{\sum _ { n = 2 } ^ { \infty } \left[\left(\frac{R_{\oplus}}{r}\right)^{n} J_{n} P_{n 0}(\cos \theta)+\right.\right. \\
& \sum_{m=1}^{n}\left(\frac{R_{\oplus}}{r}\right)^{n}\left(C_{n m} \cos m \phi+\right. \\
& \left.\left.\left.S_{n m} \sin m \phi\right) P_{n m}(\cos \theta)\right]\right\} .
\end{aligned}
$$

Here, $R_{\oplus}$ is the radius of the Earth, $J_{n}$ are the zonal harmonic coefficients, $P_{n m}$ are Legendre polynomials, and $C_{n m}$ and $S_{n m}$ are tesseral harmonic coefficients for $n \neq m$ and sectoral harmonics for $n=m$ [4]. The gravitational perturbation by the Sun and the Moon can be calculated as third-body influence

$$
\boldsymbol{a}_{\mathrm{pert}}=-\boldsymbol{d} \frac{\mu}{|\boldsymbol{d}|^{3}}
$$

for each celestial body considered, where $\boldsymbol{d}$ is the distance vector between the spacecraft and the celestial body [5]. The total acceleration on a spacecraft is simply the sum of all these influences.

The attitude of a spacecraft is also influenced by gravitation (gravity gradient) but further more by atmospheric drag, Earth's magnetic field interaction and solar radiation pressure.

\begin{tabular}{llll}
\hline & \multicolumn{3}{l}{ Used mathematical model in element } \\
Output & Sun & Earth & Moon \\
\hline posECI & f(time) & 0 & f(time) \\
oriECI & - & f(time) & - \\
gravAcc & - & eq. ( 11) & - \\
pertAcc & eq. ( 13) & eq. ( 12) & eq. ( 13 ) \\
pertTorq & - & $\mu_{\oplus}$ & - \\
atDens & - & Gill & - \\
solFlux & SMAD & - & - \\
albedoFlux & - & SMAD & - \\
infraFlux & - & SMAD & - \\
magFlux & - & simple dipole / - \\
& & IGRF 10 & \\
\hline
\end{tabular}

Table 1: Mathematical models used in the different celestial body elements. 
The effect of gravity gradient perturbation is covered by the causing gravitational parameter $\mu$. The calculation of the resulting torque is done inside the spacecraft model. The other influences of the environment on the spacecraft are given by the atmospheric density [6], the magnetic field flux [7], and the radiation (solar and infrared) at the spacecraft's position [8].

Solar and albedo radiation influences the power generation of solar cells. These two together with the infrared radiation also affect the thermal behavior of the satellite.

Table 1 shows the mapping of the celestial body outputs to the corresponding model used to calculate the physical effect.

\section{Spacecraft System}

The spacecraft system element comprises all parameters, states, interfaces and methods that are unique to the system as a whole. States of the spacecraft system, like position, velocity and so on, also apply to all elements, the system is composed of. The system hierarchy is used to determine all physical properties on system level. Thus e.g. the total mass of the spacecraft is calculated by the sum of all its component masses.

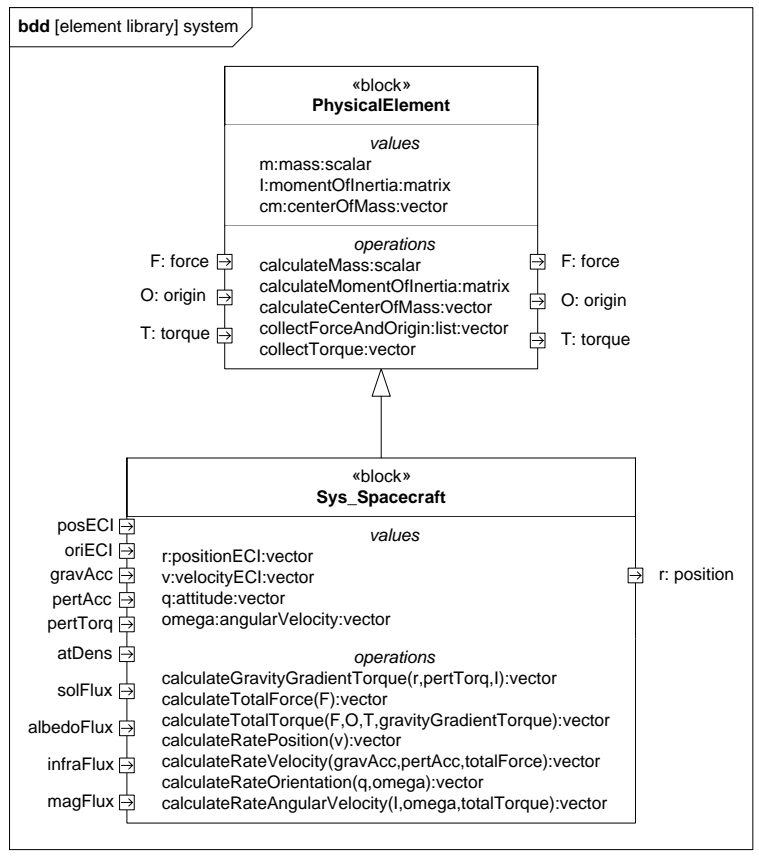

Fig. 5: Definition of the spacecraft system element class.

The rate of change of position, velocity, attitude and angular velocity is determined according to equations ( 1 ) to ( 4 ). The total external acceleration $\boldsymbol{a}_{\text {extern }}$ is given by

$$
\boldsymbol{a}_{\text {extern }}=\boldsymbol{a}_{\text {grav }}+\boldsymbol{a}_{\text {pert }} .
$$

The total torque on the spacecraft system $\mathrm{T}_{\text {total }}$ is given by

$$
\mathrm{T}_{\text {total }}=\sum \boldsymbol{T}_{i}+\sum\left(\boldsymbol{F}_{j} \times \boldsymbol{O}_{j}\right)+\boldsymbol{T}_{g g} .
$$

Here $\boldsymbol{T}_{i}$ are all input torques, $\boldsymbol{F}_{j}$ are all input forces and $\boldsymbol{O}_{j}$ the corresponding origin vector, pointing from the center of mass to the origin of the force vector. $\boldsymbol{T}_{g g}$ is the gravity gradient torque determined by

$$
\boldsymbol{T}_{\mathrm{gg}}=\frac{3 \mu}{r^{5}}[\boldsymbol{r} \times(\boldsymbol{I} \cdot \boldsymbol{r})] .
$$

Interfaces exist in order to connect the spacecraft system with the environment. The complete definition of the spacecraft system can be seen in Fig. 5 .

\section{$\underline{\text { Subsystems }}$}

The spacecraft's subsystems usually have no function themselves; they serve as an abstract container to accumulate devices and equipment which together fulfill the function. Also in the system model, subsystems serve as container, where devices can be assigned to, just to keep a clear view.

The only exception in the system model is made for the structure subsystem. This subsystem serves as the attachment point for all devices in the system. Forces and torques produced by devices are transferred to the structure subsystem. This resembles the fact that hardware is attached to the structure in reality. The total force and torque is transferred to the system level where it is evaluated further.

\section{Devices}

The functionality of the spacecraft system is enabled by the devices the spacecraft is composed of. This chapter will describe the models of the devices used in the example scenario. Each device is assigned to a subsystem according to the functionality it provides.

\section{Attitude}

The attitude of a spacecraft system is altered by the devices grouped into the attitude control subsystem (ACS). For nanosatellites this is often done by passive magnetic attitude stabilization (PMAS) [9].

For this a permanent magnet is placed inside the satellite which interferes with Earth's magnetic field, producing a vague control torque. Oscillations can be damped by hysteresis magnets placed perpendicular to the permanent magnet. The attitude about the local magnetic field vector of Earth's field cannot be influenced.

The torque produced by a magnet is calculated by

$$
\boldsymbol{T}_{\mathrm{mag}}=\boldsymbol{m} \times \boldsymbol{B}_{\mathrm{ext}}
$$


here $\boldsymbol{B}_{\text {ext }}$ is Earth's magnetic field flux and $\boldsymbol{m}$ the magnetic dipole of the magnet given by

$$
\boldsymbol{m}=\boldsymbol{M} V
$$

with the volume of the magnetic material $V$. The Magnetization $\boldsymbol{M}$ of the magnetic material is the result of the external magnetic field and the material properties.

$$
\boldsymbol{M}=\frac{\boldsymbol{B}_{\mathrm{mat}}}{\mu_{0}}-\boldsymbol{H}_{\mathrm{ext}}
$$

The magnetic flux density $\boldsymbol{B}_{\text {mat }}$ of the magnetic material is altered by the external magnetic field $\boldsymbol{H}_{\text {ext }}=\frac{\boldsymbol{B}_{\text {ext }}}{\mu_{0}}$. The value for the magnetic flux density is given by the $\mathrm{B}-\mathrm{H}$ curve of the magnetic material. The magnetic field strength in low Earth orbit is about up to $30 \mathrm{~A} / \mathrm{m}$, which covers the complete saturation cycle of a hysteresis material shown in Fig. 6 , if the material is completely rotated in the external magnetic field. The magnetic flux density of a permanent magnet is hardly altered by this external field, due to the high coercivity value of the material.

\section{B-H curve of hysteresis material}

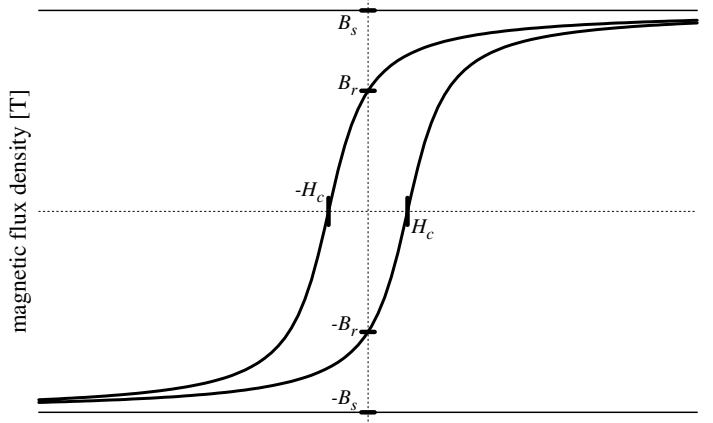

magnetic field strength $[\mathrm{A} / \mathrm{m}]$

Fig. 6: Saturation loop of a hysteresis material.

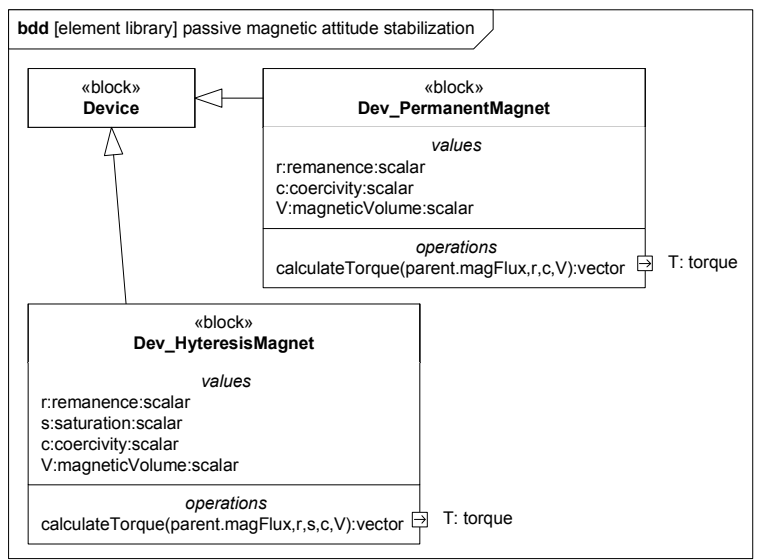

Fig. 7: Definition of the magnet device element classes.
The hysteresis characteristic is modeled with an approximation developed by Tellinen [10] which is also able to simulate minor loops. The model determines the change of magnetic flux density $b$ in the direction of the longitudinal axis of the hysteresis rod due to the applied effective external magnetic field strength $h$ (the effective field strength $h$ is the projection of the external field onto the longitudinal axis of the hysteresis material). For increasing external field strength $(d h>0)$ the change in magnetic flux density is given by

$$
\frac{d b}{d h}=\mu_{0}+\frac{b_{h-}(h)-b}{b_{h-}(h)-b_{h+}(h)}\left[\frac{d b_{h+}}{d h}(h)-\mu_{0}\right]
$$

and for decreasing external field strength $(\mathrm{dh}<0)$

$$
\frac{d b}{d h}=\mu_{0}+\frac{b-b_{h-}(h)}{b_{h-}(h)-b_{h+}(h)}\left[\frac{d b_{h-}}{d h}(h)-\mu_{0}\right] .
$$

Here $\mu_{0}$ is the permeability of vacuum. The slope of $b(h)$ is approximated by an inverse tangent function resulting in the rising slope

$$
b_{h+}(h)=2 \frac{B_{S}}{\pi} \arctan \left(\left(h-H_{c}\right) \frac{1}{H_{C}} \tan \left(\frac{B_{r}}{B_{S}} \frac{\pi}{2}\right)\right)
$$

and the falling slope

$$
b_{h-}(h)=2 \frac{B_{S}}{\pi} \arctan \left(\left(h+H_{c}\right) \frac{1}{H_{C}} \tan \left(\frac{B_{r}}{B_{S}} \frac{\pi}{2}\right)\right)
$$

The derivatives are:

$$
\begin{gathered}
\frac{d b_{h+}}{d h}(h)= \\
2 \frac{B_{S}}{\pi} \frac{1}{1+\left(\left(h-H_{C}\right) \frac{1}{H_{C}} \tan \left(\frac{B_{r} \pi}{B_{S} 2}\right)\right)^{2}} \frac{1}{H_{C}} \tan \left(\frac{B_{r}}{B_{S}} \frac{\pi}{2}\right) \\
2 \frac{d b_{h-}}{d h}(h)= \\
\pi \frac{B_{S}}{1+\left(\left(h+H_{C}\right) \frac{1}{H_{C}} \tan \left(\frac{B_{r} \pi}{B_{S} 2}\right)\right)^{2}} \frac{1}{H_{C}} \tan \left(\frac{B_{r}}{B_{S}} \frac{\pi}{2}\right) .
\end{gathered}
$$

Here $B_{S}$ is the saturation flux density, $B_{r}$ the remanence and $H_{c}$ the coercive force of the magnetic material.

\section{Power}

The power subsystem of the investigated nanosatellites is composed of solar cells arranged to small arrays, a power conditioning unit and mostly a battery. For a first analysis of the power behavior of the satellite it is sufficient to look into the power generation of the solar arrays. The power output of a solar array is

$$
P_{\mathrm{el}}=\frac{S}{s_{\odot}} n \cdot U(T) \cdot I(T) .
$$

Here $S$ is the incoming solar flux, composed of solar radiation (dependant on the sun-satellite distance) and albedo radiation, $S_{\odot}$ is the solar constant (1368 $\left.\mathrm{W} / \mathrm{m}^{2}\right), n$ is the number of solar cells in the array and $U$ are the voltage and $I$ the current of the solar cell. It 
is assumed that the solar cells work in the maximum power point. Voltage and current are dependent on the temperature of the solar cell resulting in

$$
\begin{gathered}
U(T)=U_{\mathrm{MP}}+\frac{d U_{\mathrm{MP}}}{d T}\left(T-T_{0}\right) \\
I(T)=I_{\mathrm{MP}}+\frac{d I_{\mathrm{MP}}}{d T}\left(T-T_{0}\right)
\end{gathered}
$$

with $U_{\mathrm{MP}}$ as the voltage and $I_{\mathrm{MP}}$ as the current at maximum power point, $\frac{d U_{\mathrm{MP}}}{d T}$ and $\frac{d I_{\mathrm{MP}}}{d T}$ are the temperature coefficients, $T$ is the actual array temperature and $T_{0}$ is the temperature basis for the coefficients.

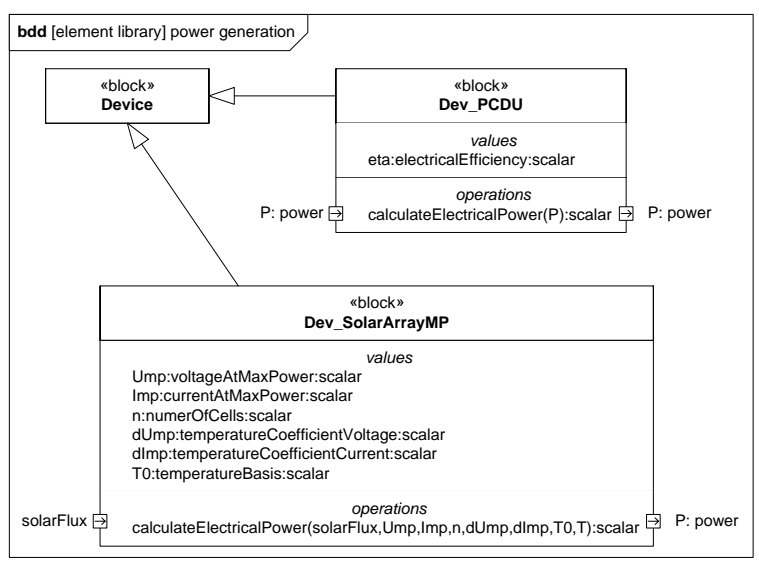

Fig. 8: Definition of the solar array device element class.

\section{$\underline{\text { Abstract Elements }}$}

Besides the physical elements described so far, some items not related to a physical representation of the real world exist. These abstract elements are used to give additional functionality in the system model or to hold parameters which are not related to a certain physical object.

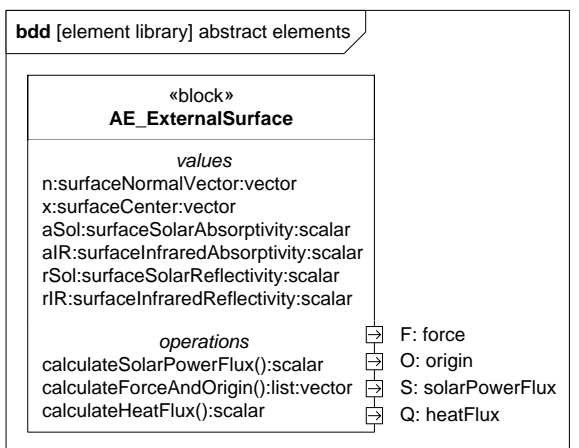

Fig. 9: Definition of the abstract element class external surface.

The abstract element presented here is the external surface element class. This provides additional properties and interfaces to already existing physical elements. The external surface element class serves as an interface between system elements and the environment. It is responsible for the calculation of solar pressure force and atmospheric drag. It calculates the solar flux on the surface dependent on the orientation of the surface and the heat flux coming from and radiated to the environment.

\section{COMPUTATIONAL SIMULATION}

The abstract and platform independent model described in the previous chapter has to be transformed into an executable form to run a simulation. The transformation is dependent on the target platform. In this case the target platform is a simulation framework implemented in $\mathrm{C}++$. Other target platforms like MatLab/Simulink are possible.

The simulator is part of the simulation framework and should be independent of the particular system model it simulates. Simulator and system model are integrated to perform the system simulation. The simulator takes care of the execution of all functionalities of the system model and the communication between the elements in the model. It propagates the states of the system elements through time with the help of an integrator. It also serves as the man-machine interface to control and monitor the simulation run.

In a first step the modeled element classes have to be translated into a $\mathrm{C}++$ implementation. This can be done in a near automated way by code generation techniques out of the SysML representations. The code generator implements the structure (i.e. parameter declarations, method calls) of the element in a way which the simulator can understand. It also implements services that are needed by the simulator to enable communication. This translation process produces a $\mathrm{C}++$ class library of elements which can be used in the simulation framework.

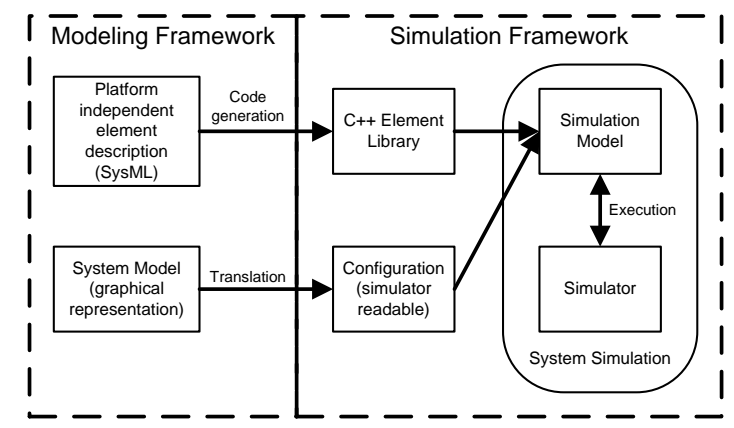

Fig. 10: Workflow for the generation of a system model and its simulation.

The next step towards a system simulation is to build a system model from the already defined element library. This also can be done graphically 
with the help of SysML diagrams [2]. These diagrams can be translated into a configuration file which can read in by the simulator. The simulator builds the simulation model from the $\mathrm{C}++$ element classes in the library according to the configuration file. Afterwards the simulation can start and the simulation model is executed.

The complete workflow from the element description until the simulation model can be seen in Fig. 10.

During simulation the simulator calls the methods of the simulation model in an iterating cycle. If an element has a dynamic state variable, the simulator calls for the derivative of this state variable. After the derivatives of all element state variables are collected the simulator integrates the state variables simultaneously and propagates the new state through the complete simulation model. The integration method can be chosen between an explicit Euler method and a Runge-Kutta $4^{\text {th }}$ order method.

\section{$\underline{\text { APPLICATION }}$}

To evaluate the modular modeling process and the simulator results the nanosatellite project Delfi-C ${ }^{3}$ from Delft University of Technology [11] was chosen as a case study. In [12] most of the information needed to set up a system model was given. The Delfi- $\mathrm{C}^{3}$ satellite is a triple CubeSat configuration with four deployable solar panels and passive magnetic attitude stabilization. The performance of the power generation and the PMAS is the subject of a system simulation.

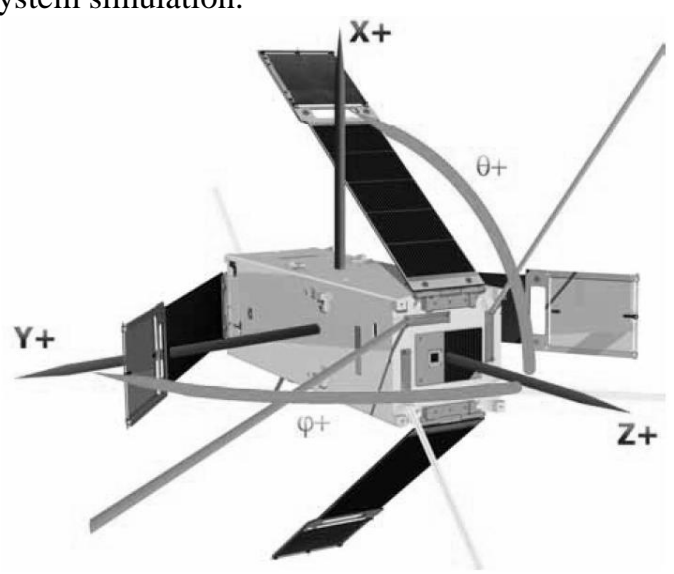

Fig. 11: Configuration of the Delfi-C ${ }^{3}$ satellite in orbit and the applied coordinate system [12].

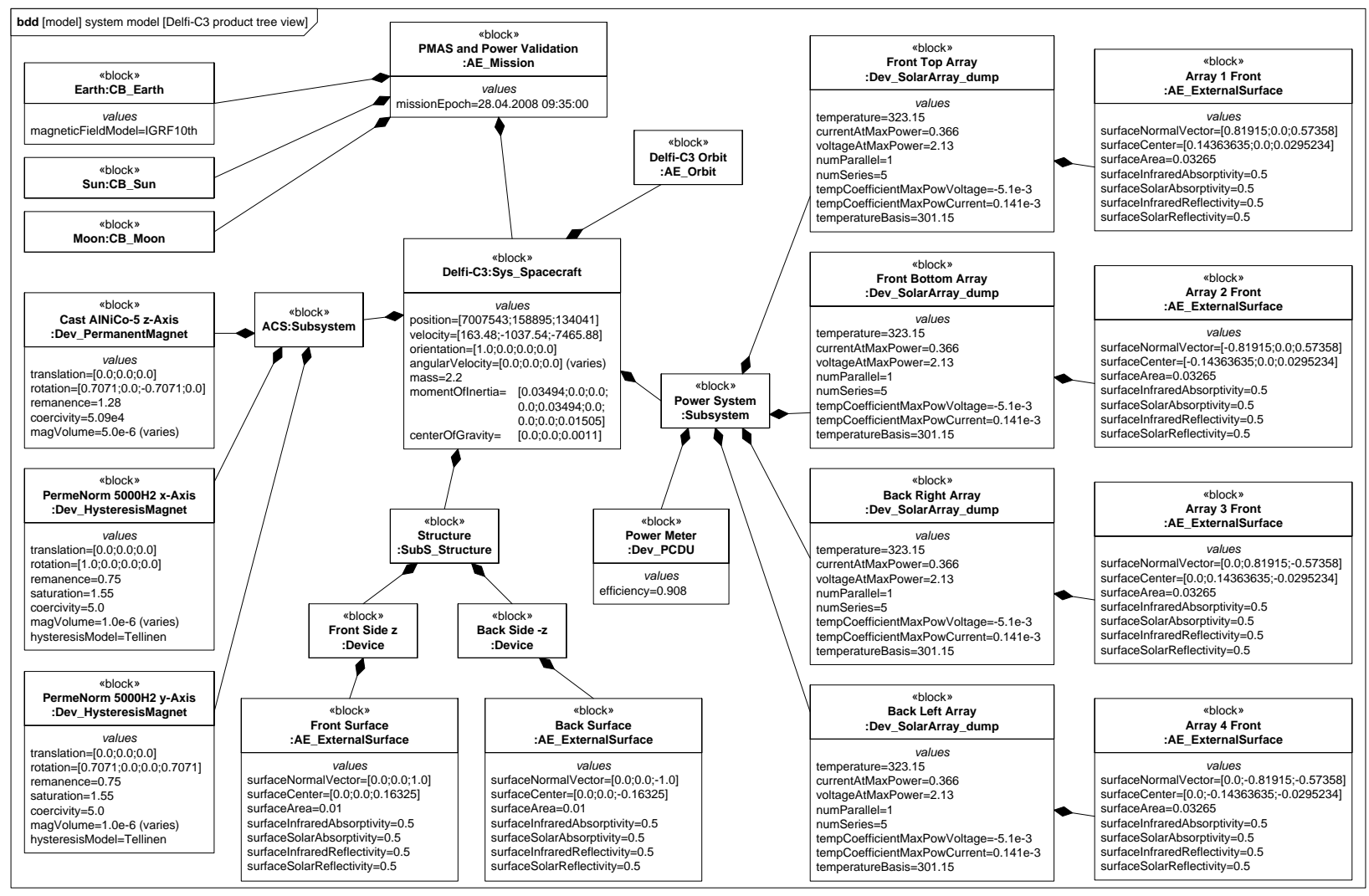

Fig. 12: Product tree view on the system model of the Delfi- $\mathrm{C}^{3}$ satellite to simulate the attitude and power generation behavior. 
The satellite was launched on 28.04.2008 and injected into a sun synchronous orbit with a height of $630 \mathrm{~km}$. The initial rotational rate of 5.1 degree per second was damped to 0.7 degree per second after two and a half months of operation (see Fig. 13 for measured values). This corresponds to a depression of $0.05714 \mathrm{deg} / \mathrm{sec}$ per day or approximately 0.00386 $\mathrm{deg} / \mathrm{sec}$ per orbit. The actual power output of the solar array is between $2.4 \mathrm{~W}$ at minimum [11] and about $5 \mathrm{~W}$ at maximum.

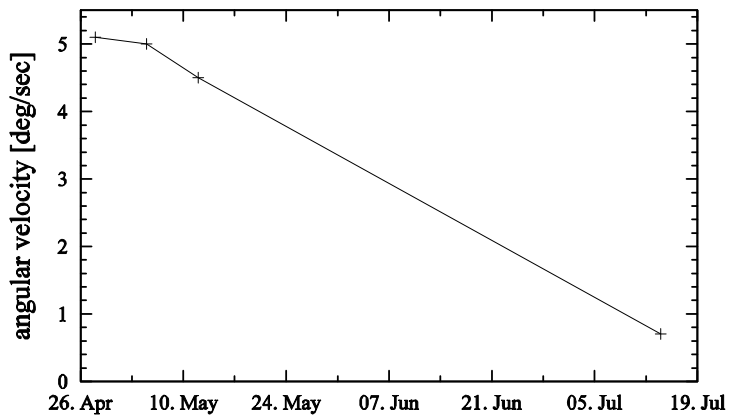

Fig. 13: Measured data on the rotational rate of Delfi$\mathrm{C}^{3}$.

The system model for the simulation is build upon very few basic element classes. The satellite system consists of a structure, an attitude control subsystem (ACS), and a power subsystem. The ACS is composed of a permanent magnet and two hysteresis magnets. The power subsystem comprises the four deployed solar panels with the solar cells, and a power meter, determining the total power generated by all solar panels. The physical properties of the satellite and its components as well as the initial conditions of all system states can be seen in the product tree view system model diagram (see Fig. 12). The satellite's coordinate system origin is located in the geometrical center of its body. The center of mass has a small deviation in z-direction.

The interaction of the system model elements is described in internal block diagrams showing the item flows between the elements. To keep a level of clarity in the modeling process, different views on the system model can be used, each showing a related functionality. Fig. 14 shows the mechanical view combining all item flows concerning the kinetic and attitude behavior of the satellite. Fig. 15 shows the electric view, combining all item flows concerned with the generation of electrical power.

Solar and atmospheric pressure forces and torques are calculated by taking only the solar panels, the front side and the back side of the satellite into account. The deployed solar panels act as side walls of the satellite and only the front sides of the solar panels contribute to the calculations. A small calculation error appears, as the surfaces of the spacecraft body sides and the backsides of the panels are neglected.

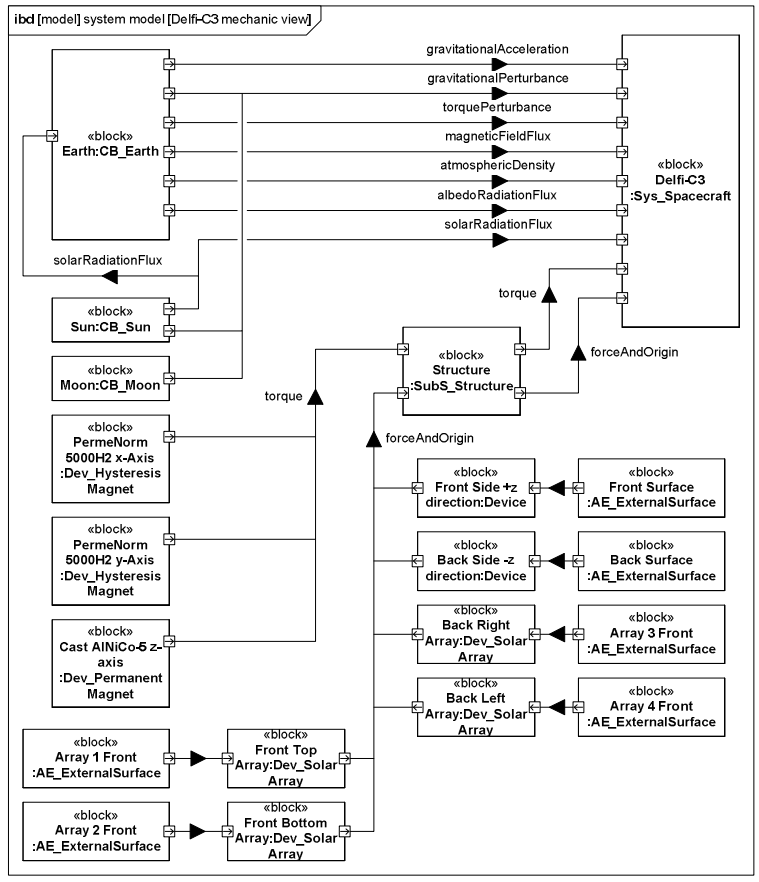

Fig. 14: Mechanic view on the system model.

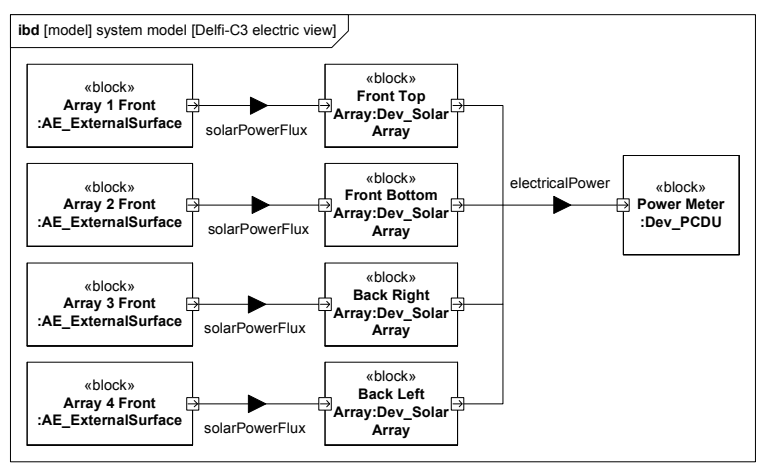

Fig. 15: Electric view on the system model

Solar power flux on the solar arrays is composed of direct solar flux plus albedo flux. Each solar panel calculates the maximum power point power output considering all external effects. Temperature effects are taken into account but a constant temperature of $323.15 \mathrm{~K}\left(50{ }^{\circ} \mathrm{C}\right)$ is used. A power meter device, which simulates the voltage regulator, is responsible for the measuring of all electrical power outputs of the panels.

\section{Attitude Behavior}

The total torque on the system is primarily accounted by the permanent magnet. The magnitude of the total torque is about $5 \cdot 10^{-5} \mathrm{Nm}$ at maximum. The 
contribution of all other disturbances, aside from the magnetic moment, sums up to some $10^{-7} \mathrm{Nm}$.

Simulation with varying initial conditions show that magnetic lock is achieved within a few orbits. Residual oscillations are present about all axes which are slowly damped by the hysteresis magnets.

Both magnetic materials, the permanent magnet and the hysteresis magnet, contribute to the total system magnetic moment vector and thus to the orientation of the alignment axis [9]. The angle between the permanent magnet axis and the magnetic field lines, which can be seen as the alignment error is dependent on the ratio of the magnetic moment contribution by the permanent and hysteresis magnets. Since the magnetic moment is proportional to the amount of magnetic material, the alignment error is dependent on the mass or volume ratio of the materials. A tradeoff has to be made to find the right ratio. With almost no hysteresis material present, the alignment error can be reduced to almost zero. In this case the damping capability is no longer granted and the residual oscillation will last for a long time.

\section{PMAS setup 1}

In the first system model setup, the volume of the permanent magnet is set to $5 \cdot 10^{-6} \mathrm{~m}^{3}$, the volume of the hysteresis material is $1 \cdot 10^{-7} \mathrm{~m}^{3}$ on each perpendicular axis. Fig. 18 to Fig. 18 show the prints of the alignment of the permanent magnet axis ( $\mathrm{z}$-axis of the satellite body) to the magnetic field lines and to the Nadir vector for the three different initial conditions listed in Table 2 from top to bottom. The initial orientation of the spacecraft was aligned to the ECI coordinate system.

\begin{tabular}{ll}
\hline Scenario & Initial angular velocity vector \\
\hline Steady & $(0|0| 0)^{\mathrm{T}}$ \\
Rotation z & $(0|0| 0.175)^{\mathrm{T}}$ \\
Rotation $\mathrm{x}$ & $(0.175|0| 0)^{\mathrm{T}}$ \\
\hline
\end{tabular}

Table 2: Scenarios and initial conditions.

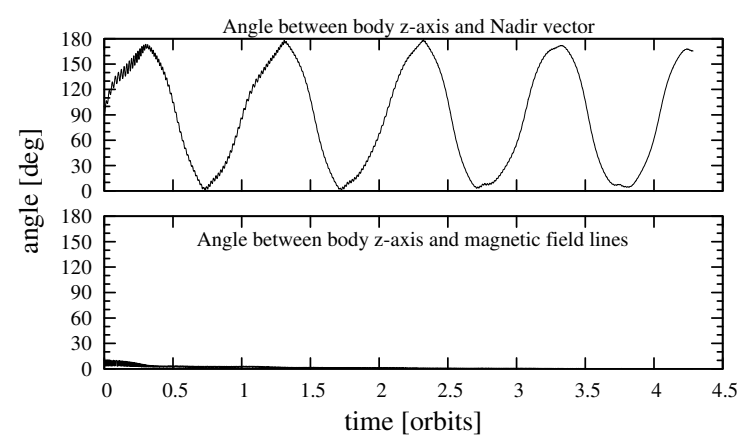

Fig. 16: Alignment of the body z-axis for the steady scenario. $V_{\text {hyst }}=1 \cdot 10^{-7}$ on $\mathrm{x}^{-}$and $\mathrm{y}$-axis; $V_{\text {mag }}=5 \cdot 10^{-6}$ on z-axis.

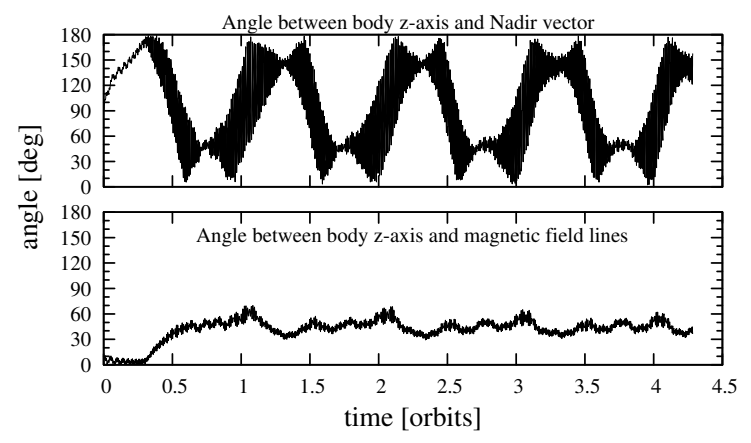

Fig. 17: Rotation z scenario.

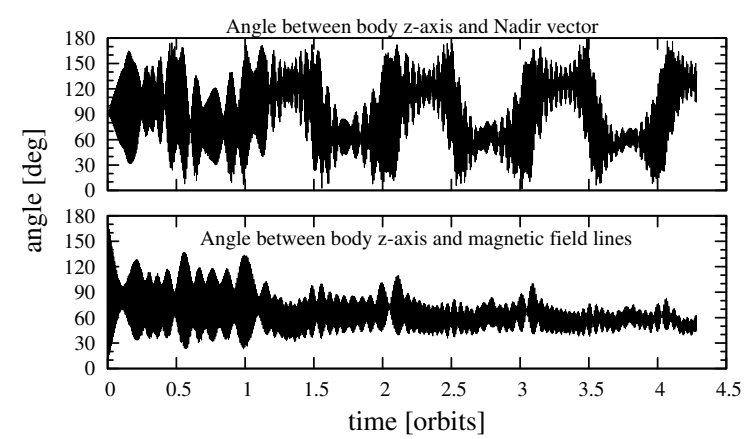

Fig. 18: Rotation x scenario.

In the first diagram one can see a perfect alignment to the magnetic field lines. The satellite flips twice an orbit at the poles in each case. The second diagram also shows a magnetic lock of the satellite, but due to the initial angular velocity about the $\mathrm{z}$-axis and the mass distribution, the satellite nutates. This results in a deviation of the body axes to the magnetic field axis of about $45^{\circ}$. Also strong residual oscillations are present which are hardly damped. The same effect can be seen in the third diagram. Magnetic lock is established after one orbit, the deviation between body $\mathrm{z}$-axis and magnetic field lines is about $50^{\circ}$.

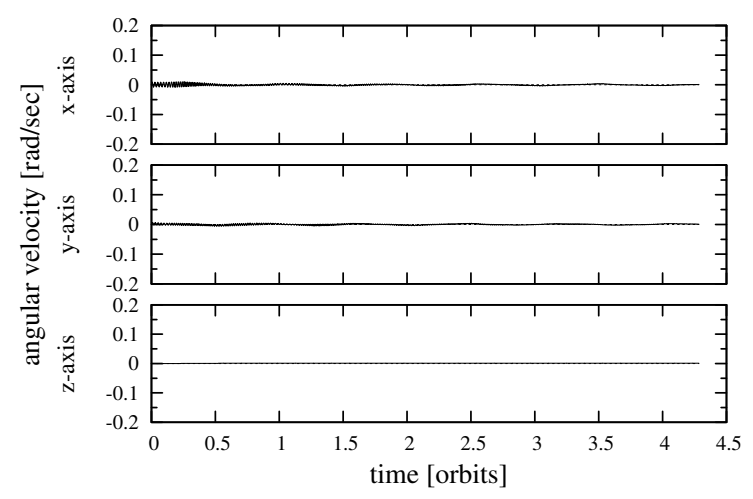

Fig. 19: Angular velocity response for steady scenario. 


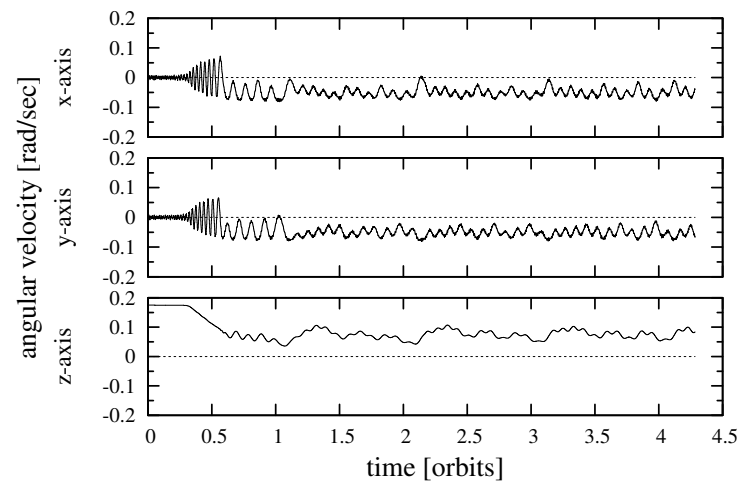

Fig. 20: Rotation z scenario.

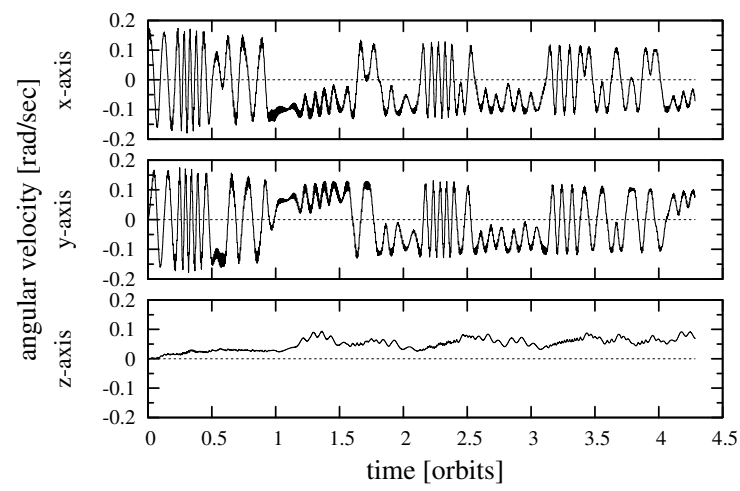

Fig. 21: Rotation x scenario.

The oscillations can be seen in Fig. 19 to Fig. 21 showing the progress of the angular velocities. The steady scenario has almost no angular velocity present. In the rotating-z scenarios the angular velocity about the $\mathrm{z}$-axis is exchanged to the other body axes after a short period of time. This is correlated with the magnetic lock. Afterwards the angular velocities stay relatively constant. The bottom diagram shows the other way around. The angular velocity is quickly distributed over the $\mathrm{x}$ - and $y$-axis and some of the angular velocity is transferred to the z-axis. The dampening effect is very weak in all cases. Fig. 22 shows the magnitude of the angular velocity vector for the rotation- $x$ scenario. The decrease in angular velocity is about $0.1653 \mathrm{deg} / \mathrm{sec}$ per orbit.

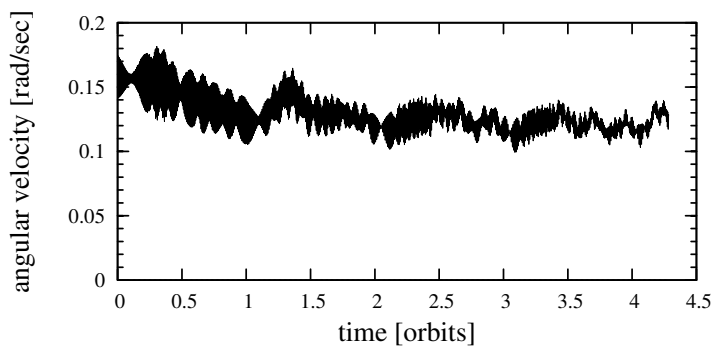

Fig. 22: Magnitude of the angular velocity vector, showing the small dampening effect.

\section{PMAS setup 2}

The arbitrary volume of the magnetic materials in the previous setup is now changed to the real values of Delfi- $\mathrm{C}^{3}$. The volume of the permanent magnet is $2.977 \cdot 10^{-7} \mathrm{~m}^{3}$, the volume of the hysteresis material is $7.69 \cdot 10^{-7} \mathrm{~m}^{3}$ on each perpendicular axis. This is a much different ratio between the two materials, less permanent magnet and more hysteresis material. The satellite is supposed to align not so well to the magnetic field lines. This is desired by the system design because the two experimental payloads of Delfi- $\mathrm{C}^{3}$, a sun sensor and thin film solar cells, should be lighted from different angles. Therefore the attitude control system is supposed to not align well with the magnetic field lines but mainly to dampen excessive rotational rates.

The magnetic alignment for the steady scenario is shown in Fig. 23. The axis of the permanent magnet is no longer perfectly aligned to the magnetic field lines. The deviation is about $80^{\circ}$. Small oscillations are present which are slowly damped.

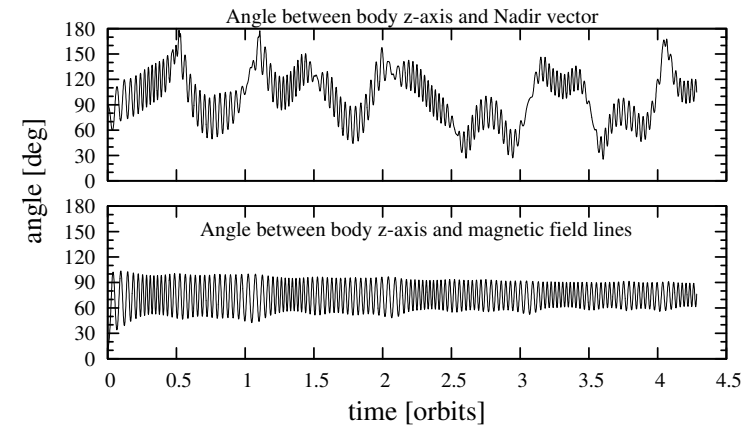

Fig. 23: Alignment for the steady scenario.

The system response for rotating initial conditions is very similar (see Fig. 24 and Fig. 25). After one orbit, magnetic lock is achieved. Residual oscillations are damped.

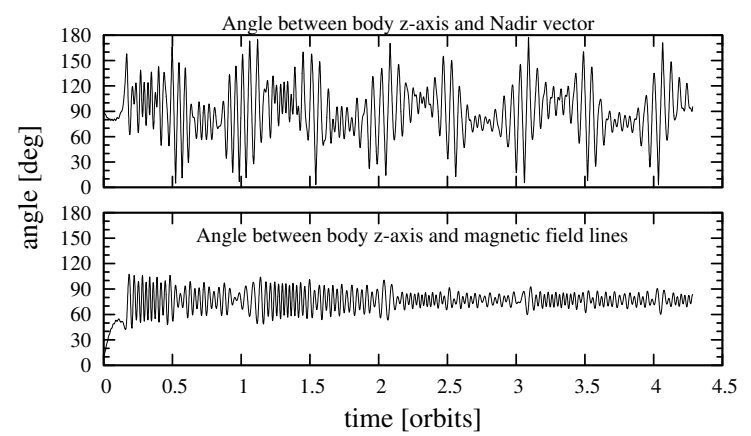

Fig. 24: Alignment for the rotation z scenario. 


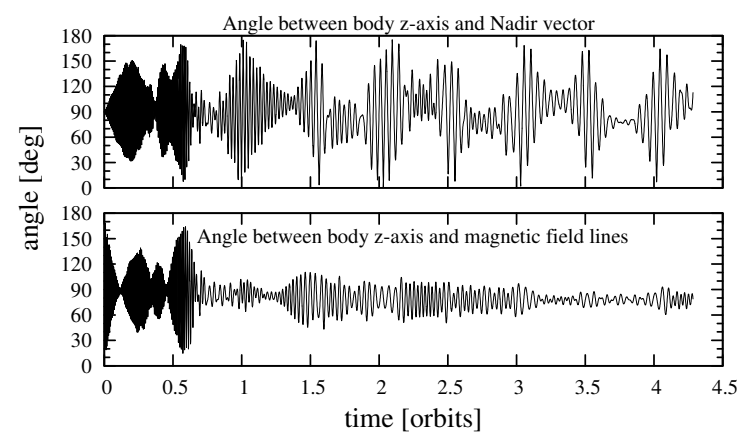

Fig. 25: Alignment for the rotation x scenario.

The dampening effect is shown in Fig. 26 to Fig. 27.

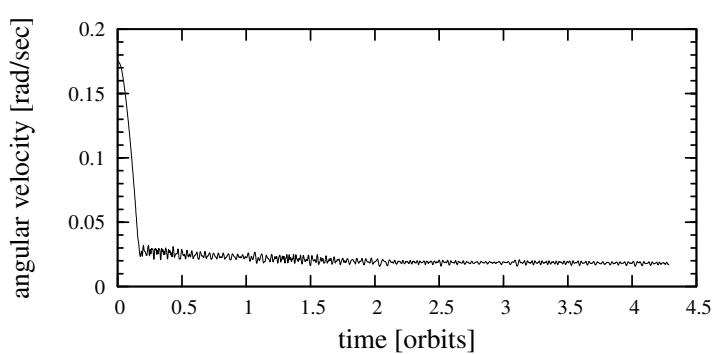

Fig. 26: Magnitude of the angular velocity vector for rotation z scenario.

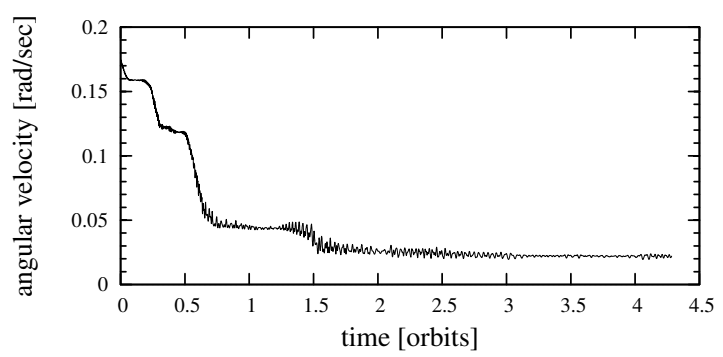

Fig. 27: Magnitude of the angular velocity vector for rotation $\mathrm{x}$ scenario.

\section{Hysteresis correction}

As it is stated in [13], the hysteresis material does not behave as theory predicts. A much weaker influence of the hysteresis material on the angular velocity is observed in experiments. For this reason a correction factor is introduced into the hysteresis model. The correction factor decreases the effective magnetic field strength of the external field on the hysteresis material. This results in a lower magnetization of the material.

With a correction factor of 50 the area of the hysteresis cycle decreases significantly. The Earth magnetic field is no longer able to saturate the hysteresis material. Fig. 28 to Fig. 30 show the cycle of a hysteresis rod during simulation with a correction factor of 0,50 , and 100 , respectively. The complete saturated loop in the first case is reduced to much smaller minor loops in the latter cases. This surely results in a weaker dampening effect of the hysteresis rods.

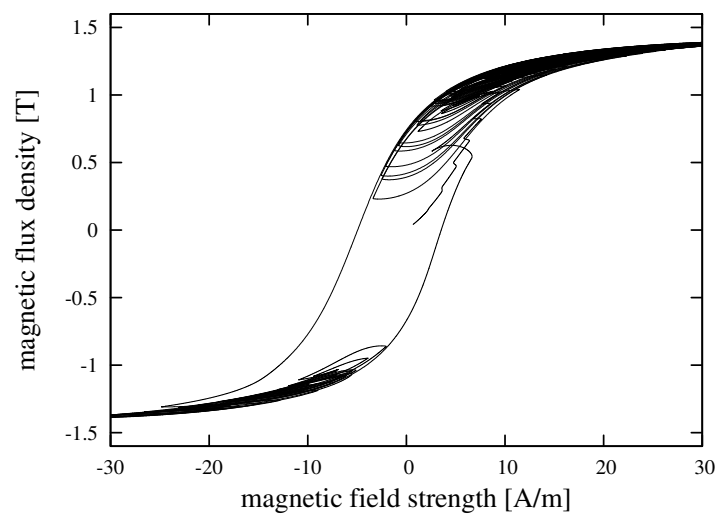

Fig. 28: Hysteresis curve with no correction factor. Saturation and minor loops can be seen.

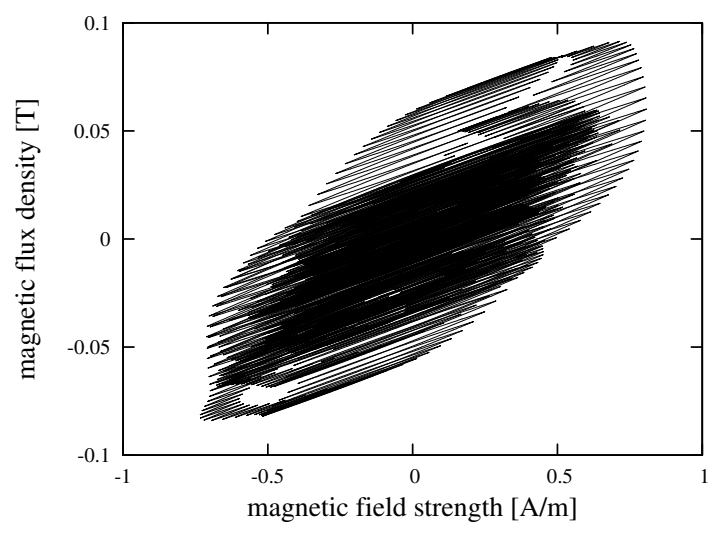

Fig. 29: Hysteresis curve with correction factor 50. Only minor loops are present.

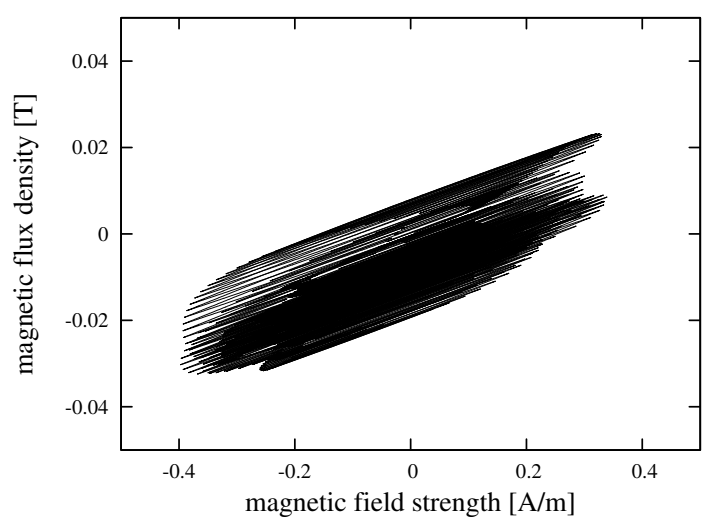

Fig. 30: Hysteresis curve with correction factor 100 . Only minor loops are present.

The corresponding depression in angular velocity can be seen in Fig. 31 to Fig. 33. These plots can be compared to the previous ones but have a much 
longer simulated duration of 26 orbits. The initial condition is the same as in the rotation- $\mathrm{X}$ scenario.

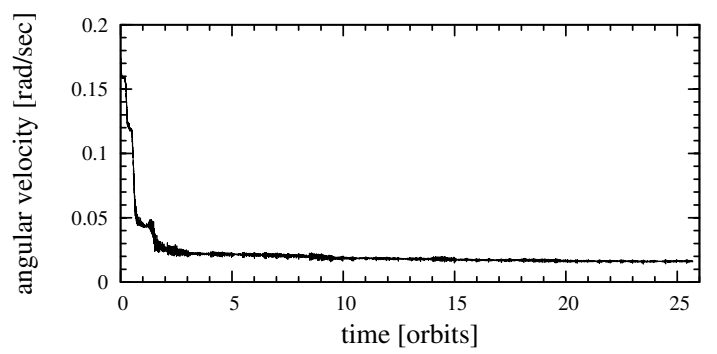

Fig. 31: Angular velocity depression with a hysteresis correction factor of 0 .

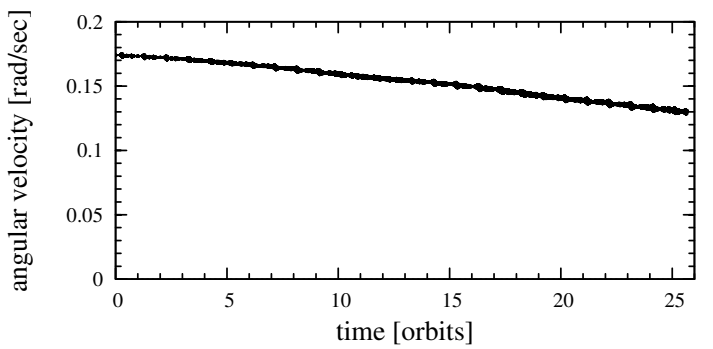

Fig. 32: Angular velocity depression with a hysteresis correction factor of 50 .

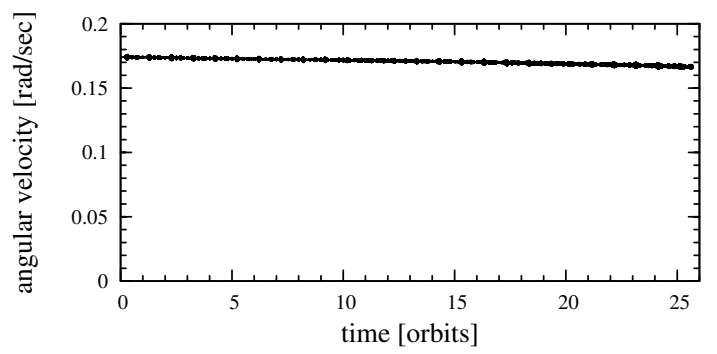

Fig. 33: Angular velocity depression with a hysteresis correction factor of 100 .

In the first plot a massive decay to about 0.02 $\mathrm{rad} / \mathrm{sec}$ can be seen over the first two orbits going into a very shallow decrease of $0.0136 \mathrm{deg} / \mathrm{sec}$ per orbit. The second graph shows an almost constant depression of $0.1089 \mathrm{deg} / \mathrm{sec}$ per orbit over the complete simulated time. The third graph shows a constant depression of $0.0145 \mathrm{deg} / \mathrm{sec}$ per orbit over the complete simulated time.

\section{$\underline{\text { Power Behavior }}$}

The power generation is mostly independent on the magnetic stabilization configuration. Fig. 34 shows the generated power for a fast rotating scenario and a steady scenario. Both diagrams show an average power generation of about $3.2 \mathrm{~W}$. This value is given for an efficiency of the voltage regulator of $90.8 \%$. Taking into account a total efficiency of the power generation string of about $75 \%$, the generated power can drop to $2.4 \mathrm{~W}$. This is in accordance with the in orbit measurements.
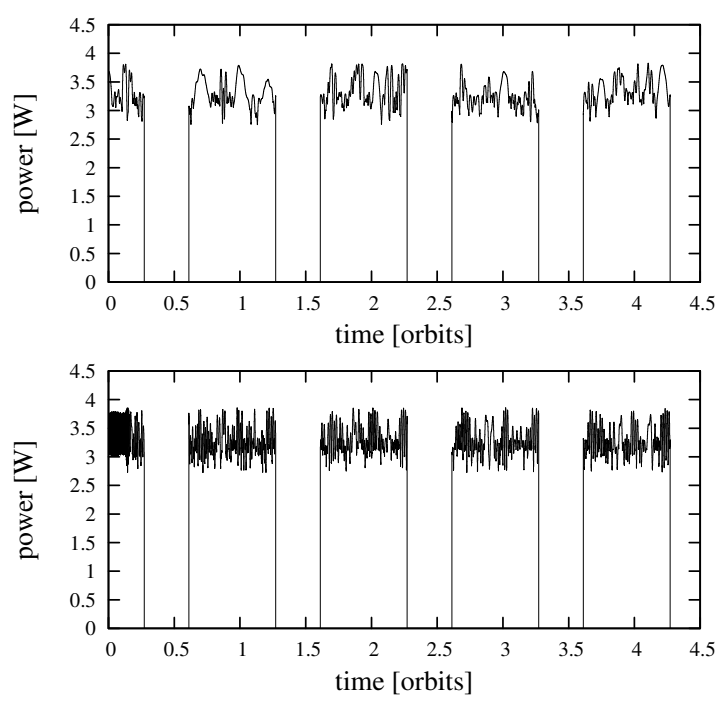

Fig. 34: Generated power for steady (top) and rotating (bottom) initial conditions.

\section{CONCLUSION}

System simulation can contribute to the development cycle of a satellite project. The presented modeling method utilizes a very modular and adaptive approach. There are many similarities to the concurrent engineering approach. A central model of the system is present on which different engineering disciplines can work concurrently. The system model described here holds data on the structure and the dynamic behavior of the spacecraft system. With a growing library of building blocks one can easily create a system model out of the box and run dynamic simulations. The dynamic system simulation can be used to size critical design parameters, analyze the system response under varying conditions, and to verify the system performance.

In an application scenario, the complete approach to perform a system simulation was used. It has been shown that a simulation of a complete system is feasible and can be built by modular model building blocks. The dynamic attitude and power behavior of the nanosatellite project Defli- $\mathrm{C}^{3}$ has been simulated. The interactions between orbit, attitude and power behavior were included. The next step is to complete the system model and include thermal and communication behavior.

However the attitude behavior of the satellite shows some discrepancies with real measurement. One has to keep in mind that a simulation is only as good as the model behind it. Some more effort has to be made to come up with a suitable hysteresis material model to improve the PMAS simulation of small satellites. 
Some hypotheses on the different behavior of hysteresis material are:

- The magnitude of the hysteresis effect does not scale linear with the size and volume.

- The saturation of the material takes more time than the rotational change, creating more minor loops.

- The influence of other magnetic material in the satellite (e.g. the permanent magnet) on the hysteresis material is much higher.

These theories have to be evaluated in future work.

\section{REFERENCES}

[1] Object Management Group, OMG Systems Modeling Language (OMG SysML $\left.{ }^{\mathrm{TM}}\right)$. Version 1.1 (2008).

[2] M. Raif, M. Brandstätter, C. Eckl, and U. Walter, A Modular Approach for Modeling and Dynamic Simulation of Spacecraft Systems. Proceedings of the 7th IAA Symposium on Small Satellites for Earth Observation, 2009.

[3] O. Montenbruck and E. Gill, Satellite Orbits. Models, Methods, and Applications (Springer, Berlin, 2005).

[4] J. R. Wertz, Spacecraft attitude determination and control (Kluwer Academic Publishers, Dordrecht, The Netherlands, 1978).

[5] D. A. Vallado and W. D. McClain, Fundamentals of Astrodynamics and Applications (Kluwer Academic Publishers, Dordrecht, 2001), Vol. 12.

[6] E. Gill, Smooth Bi-Polynominal Interpolation of Jacchia 1971 Atmospheric Densities For Efficient Satellite Drag Computation. DLRGSOC IB 96-1, 1996.

[7] J. Davis, Mathematical Modeling of Earth's Magnetic Field, Technical Note (2004).

[8] W. J. Larson and J. R. Wertz, Space Mission Analysis and Design. Third Edition (Microcosm Press, El Segundo, Calif., 1999).

[9] J.-F. Levesque, Passive Magnetic Attitude Stabilization using Hysteresis Materials. SIgMA-PU-006-UdeS, 2003.

[10] J. Tellinen, A Simple Scalar Model for Magnetic Hysteresis, IEEE Transactions on Magnetics 34, 4 (1998).

[11] W. Ubbels et al., First Flight Results of the Delfi-C3 Satellite Mission. 22nd Annual AIAA/USU Conference on Small Satellites, 2008.

[12] F. te Hennepe, B. T. Zandbergen, and R. Hamann, Simulation of the Attitude Behaviour and Available Power Profile of the DELFI-C3 Spacecraft with Application of the OPSIM Platform. 1st CEAS European Air and Space
Conference, Berlin, Germany, 10-13 September 2007, 2007.

[13] F. Poppenk, Design and Testing of an Attitude Control System for a Nano Satellite, Master Thesis Report, Delft University of Technology, 2009. 